\title{
Candidate LBVs in Local Group galaxies
}

\author{
Luis J. Corral ${ }^{1}$ and Artemio Herrero ${ }^{1,2}$ \\ ${ }^{1}$ Instituto de Astrofísica de Canarias, \\ C/Vía Láctea s/n, E-38205 La Laguna, La Laguna, España \\ ${ }^{2}$ Departamento de Astrofísica, Universidad de La Laguna, \\ Avda. Astrofísico Francisco Sánchez, s/n, E-38071 La Laguna, España
}

\begin{abstract}
We present the results of photometric searches for LBV candidates (LBVc) made in some Local Group galaxies. We found eight candidates in a southern region of M 33 with characteristics similar to Var B and Var C. In IC 10, a galaxy without any LBV or LBVc reported before, we found one candidate.
\end{abstract}

\section{Introduction}

Luminous Blue Variables (LBV) are a group of irregular variables characterized by their intrinsic high luminosities, their photometric behavior and their related spectroscopic changes. Members of this group have been identified in the Milky Way and in nearby galaxies. A review on LBVs can be found in Humphreys \& Davidson (1994) and in Bohannan (1997).

Historically these objects have been found by their variability shown in optical and UV bands, but these variations are followed by stable states with timescales of the order of decades. There are objects with the same spectral characteristics, but without any variation recorded in historical times and it is possible that some of them are LBVs in quiescent phases. These objects are known as LBV candidates (LBVc).

Balmer and $\mathrm{He} \mathrm{I}$ lines in emission are common characteristics of the spectra of LBV and LBVc. HeI $\lambda 5015.6 \AA$ usually falls in the wavelength band of the filters used to isolate the [O III] $\lambda 5007 \AA$ and sometimes objects with emission in $\mathrm{He}$ I are confused with objects with [O III] emission. If we observe a region of a galaxy with $\mathrm{H} \alpha,[\mathrm{O}$ III] and He I filters, some of the objects showing emission in all bands could turn out to be LBVc, making the search of these elusive objects less difficult.

\section{M 33, IC 10 and NGC 6822}

The southern area of M 33 was observed with NOT-ALFOSC using narrow-band filters of $\mathrm{H} \alpha,[\mathrm{O} \mathrm{III}]$ and $\mathrm{He} \mathrm{I} \lambda 5876 \AA$ as well as two adjacent continuum regions. The in-line images were subtracted by the corresponding continua adequately scaled, and the emission regions and objects detected were calibrated with ob- 
servations of standard stars observed with the same set of filters. We detect 249 emission-line objects, including the two known LBVs in the area observed: $\operatorname{Var} B$ and $\operatorname{Var} C$. Accordingly with the characteristics of $\operatorname{Var} B$ and $\operatorname{Var} C$ in this observations, we expect that LBVc have emission in all filters, with strong $\mathrm{H} \alpha$ emission and He I emission greater than [O III] emission. We found eight objects with these characteristics, the results are presented elsewhere (Corral \& Herrero in preparation).

IC 10 is a peculiar galaxy in the Local Group, it presents the highest concentration of WR stars in a nearby galaxy (Massey et al. 1995) and is believed to present a strong burst episode of star formation. There are no LBV or LBVC reported in this galaxy. We observe IC 10 using MOSCA at the 3.5m CAHA telescope with filters that isolate $\mathrm{H} \alpha,[\mathrm{O}$ III] and He I $\lambda 6755 \AA$. Using DAOPHOT in IRAF we obtain the instrumental magnitudes and, comparing the in-line with the continuum magnitudes, a preliminary analysis yields one single candidate (Corral \& Herrero in preparation.)

NGC 6822 is the third nearest irregular galaxy, after the Magellanic Clouds. It is not associated with the group of galaxies that surrounds M 31 or the Milky Way and does not have any known companion (van den Berg 1999). The metallicity is low and the content of gas makes it a moderate gas-rich galaxy (de Blok \& Walter 2000). To study this galaxy, we used the photometric criteria presented by King et al. (1998) when they found five blue objects in M 31 with strong $\mathrm{H} \alpha$ emission but without emission in the $[\mathrm{S} \mathrm{II}] \lambda \lambda 6727,6731 \AA$ lines that turn out to be characteristic for LBVc.

We observed NGC 6822 with the $1.5 \mathrm{~m}$ telescope at SPM observatory. The filters used were $\mathrm{H} \alpha$, [O III], [S II] and two nearby continua. Problems during the observations with the [S II] filter rendered these images of poor quality and we can not conclude that there are some objects with the same characteristics as King et al. objects in M 31. A byproduct of these studies is the discovery of candidate planetary nebulae (PNc). In the case of M33 we report $48 \mathrm{PNc}$ (Corral \& Herrero 2001), and in the images of NGC 6822 we detect four objects with these chracteristics.

It is true that the nature of all these objects has to be confirmed with spectroscopy, but at least we narrow the list of objects that we have to observe.

\section{References}

Bohannan, B. 1997, in: A. Nota \& H. Lamers (eds.), Luminous Blue Variables: Massive Stars in Transition, ASP-CS 120, 3

Corral, L.J., Herrero, A. 2001, A\&A 376, 820

de Blok, W.J.G., Walter, F. 2000, ApJ 537, 95

Humphreys, R., Davidson, K. 1994, PASP 106, 1025

King, N.L., Walterbos, R.A.M., Braun, R. 1998, ApJ 507, 210

Massey, P., Armandroff, T.E., Pyke, R., Patel, K., Wilson, C.D. 1995, AJ 110, 2715

van den Bergh, S. 1999 AJ 117, 2211 